\title{
Front Matter: Volume 7986
}

, "Front Matter: Volume 7986," Proc. SPIE 7986, Passive Components and Fiber-Based Devices VII, 798601 (14 March 2011); doi: 10.1117/12.890538

SPIE Event: Asia Communications and Photonics Conference and Exhibition, 2010, Shanghai, Shanghai, China 


\title{
PROCEEDINGS OF SPIE
}

\section{Passive Components and Fiber-Based Devices VII}

\author{
Perry P. Shum \\ Editor

\section{8-12 December 2010 Shanghai, China}

Cosponsored by

IEEE Photonics Society • Optical Society of America • SPIE • Chinese Optical Society China Institute of Communications

Local Organizing Committee

Fudan University • Pudong Optoelectronics Industry Association of Shanghai

Shanghai Jiao Tong University • Shanghai Institute of Optics and Fine Mechanics

Alcatel-Lucent Shanghai Bell

Best Student Paper Sponsor

Thorlabs, Inc.

Published by

SPIE • Optical Society of America • IEEE Photonics Society

Volume 7986

Proceedings of SPIE, 0277-786X, v. 7986 
The papers included in this volume were part of the technical conference cited on the cover and title page. Papers were selected and subject to review by the editors and conference program committee. Some conference presentations may not be available for publication. The papers published in these proceedings reflect the work and thoughts of the authors and are published herein as submitted. The publisher is not responsible for the validity of the information or for any outcomes resulting from reliance thereon.

Please use the following format to cite material from this book:

Author(s), "Title of Paper," in Passive Components and Fiber-Based Devices VII, edited by Perry P. Shum, Proceedings of SPIE-OSA-IEEE Asia Communications and Photonics, SPIE Vol. 7986 (SPIE, Bellingham, WA, 2011) Article CID Number.

ISSN 0277-786X

ISBN 9780819485540

Published by

SPIE

P.O. Box 10, Bellingham, Washington 98227-0010 USA

Telephone +1 3606763290 (Pacific Time) · Fax +1 3606471445

SPIE.org

Optical Society of America

2010 Massachusetts Ave., N.W., Washington, D.C., 20036 USA

Telephone +1 2022238130 (Eastern Time) · Fax +1 2022231096

OSA.org

IEEE Photonics Society

445 Hoes Lane, Piscataway, New Jersey, 08855 USA

Telephone +1 7325628434 (Eastern Time) · Fax +1 7325628434

IEEE.org

Copyright (C) 2011, Society of Photo-Optical Instrumentation Engineers, Optical Society of America, and IEEE Photonics Society.

Copying of material in this book for internal or personal use, or for the internal or personal use of specific clients, beyond the fair use provisions granted by the U.S. Copyright Law is authorized by SPIE subject to payment of copying fees. The Transactional Reporting Service base fee for this volume is $\$ 18.00$ per article (or portion thereof), which should be paid directly to the Copyright Clearance Center (CCC), 222 Rosewood Drive, Danvers, MA 01923. Payment may also be made electronically through CCC Online at copyright.com. Other copying for republication, resale, advertising or promotion, or any form of systematic or multiple reproduction of any material in this book is prohibited except with permission in writing from the publisher. The CCC fee code is $0277-786 \mathrm{X} / 11 / \$ 18.00$.

Printed in the United States of America.

Publication of record for individual papers is online in the SPIE Digital Library.

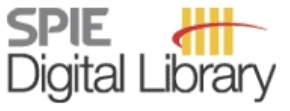

SPIEDigitalLibrary.org

Paper Numbering: Proceedings of SPIE follow an e-First publication model, with papers published first online and then in print and on CD-ROM. Papers are published as they are submitted and meet publication criteria. A unique, consistent, permanent citation identifier (CID) number is assigned to each article at the time of the first publication. Utilization of CIDs allows articles to be fully citable as soon they are published online, and connects the same identifier to all online, print, and electronic versions of the publication. SPIE uses a six-digit CID article numbering system in which:

- The first four digits correspond to the SPIE volume number.

- The last two digits indicate publication order within the volume using a Base 36 numbering system employing both numerals and letters. These two-number sets start with $00,01,02,03,04,05,06,07,08$, $09,0 \mathrm{~A}, \mathrm{OB} \ldots \mathrm{OZ}$, followed by 10-1Z, 20-2Z, etc.

The CID number appears on each page of the manuscript. The complete citation is used on the first page, and an abbreviated version on subsequent pages. Numbers in the index correspond to the last two digits of the six-digit CID number. 


\section{Contents}

ix Symposium Committees

xi Conference Committee

\section{SESSION 1 SIGNAL PROCESSING AND SYSTEM I}

798602 A switchable demodulator for both DPSK and DQPSK formats [7986-01]

D. Zhang, Huazhong Univ. of Science and Technology (China) and Accelink Technologies Co., Ltd. (China); W. Liu, Huazhong Univ. of Science and Technology (China); L. Chen, Q. Hu, Z. Xue, Accelink Technologies Co., Ltd. (China)

798603 Passive components modeling for optical network-on-chip [7986-48]

B. Wang, I. O'Connor, E. Drouard, Institut des Nanotechnologies, CNRS, Univ. de Lyon (France)

\section{SESSION 2 NANO FIBER}

798604 CD and PMD monitoring based on RF spectrum analysis with optical filtering (Invited Paper) [7986-53]

C. YU, National Univ. of Singapore (Singapore) and A*STAR (Singapore); J. Yang, National Univ. of Singapore (Singapore)

$798605 \quad$ PbSe quantum dots fiber amplifier based on sol-gel self-assembly method [7986-36]

X. Sun, Y. Dong, C. Li, X. Liu, G. Liu, L. Xie, Shanghai Univ. (China)

\section{SESSION 3 BEST STUDENT PAPER SESSION}

798606 Correlated photon pair generation in silicon wire waveguides [7986-41]

J. Cheng, Q. Zhou, W. Zhang, Y. Huang, J. Peng, Tsinghua Univ. (China)

798607 Experimental demonstration of $\pm \pi / 2$-phase-shifted SSFBG encoder for security improvement in time-spreading OCDMA [7986-04]

B. Dai, Z. Gao, X. Wang, Heriot-Watt Univ. (United Kingdom); N. Kataoka, N. Wada, National Institute of Information and Communication Technology (Japan)

798608 Silver nanowire based plasmon propagation, coupling and splitting at $1.55 \mu \mathrm{m}$ wavelength (Best Student Paper Award) [7986-15]

Q. Li, Royal Institute of Technology (Sweden); S. Wang, Zhejiang Univ. (China); Y. Chen, M. Yan, Royal Institute of Technology (Sweden); L. Tong, Zhejiang Univ. (China); M. Qiu, Royal Institute of Technology (Sweden)

798609 Fractional Talbot effect induced rational-harmonic mode-locking of $40-\mathbf{G H z}$ SOA fiber laser [7986-140]

J.-J. Kang, C.-K. Lee, National Sun Yat-Sen Univ. (Taiwan, China); G.-R. Lin, National Taiwan Univ. (Taiwan, China) 
7986 OA Optical S-R latch demonstration using injection-locked single-mode FP-LD [7986-18]

M. Rakib-Uddin, T. Q. Hoai, B. Nakarmi, Y. H. Won, KAIST (Korea, Republic of)

SESSION 4 FIBER LASER

7986 OB Ge-codoped laser fibers for mitigating stimulated Brillouin scattering in high power fiber amplifiers (Invited Paper) [7986-09]

X. Chen, M.-J. Li, A. Liu, Corning Inc. (United States)

7986 OC Dual-wavelength DFB fiber laser based on equivalent phase shift and double exposure method [7986-08]

L. Jia, L. Zhang, Z. Yin, X. Chen, Nanjing Univ. (China)

7986 OD Transient state of wavelength tuning in mode-locked lasers with a dispersive cavity [7986-05]

S. Pan, Nanjing Univ. of Aeronautics and Astronautics (China); C. Lou, Tsinghua Univ. (China)

7986 OE Numerical analysis on wavelength-switchable lasers with inhomogeneous and homogeneous line broadening media [7986-113]

S. Qin, Y. Zou, X. Zhu, Alcatel-Lucent Shanghai Bell (China)

\section{SESSION 5 OPTICAL WAVEGUIDE}

7986 OF Design and fabrication of SU-8 arrayed-waveguide gratings using multimode interference couplers [7986-43]

Y. Zhu, B. Yang, Y. Jiao, D. Dai, Zhejiang Univ. (China)

\section{SESSION 6 FILTERS AND GRATINGS}

7986 OG All-solid photonic bandgap fiber gratings (Invited Paper) [7986-44]

Y. Liu, Z. Wang, B. Tai, J. Xu, Nankai Univ. (China)

$7986 \mathrm{OH}$ Fiber-optic temperature sensing based on LPFG fabricated with heat-shrinkable tube and screw [7986-40]

Y. Tsutsumi, H. Yamamoto, Y. Miyoshi, M. Ohashi, Osaka Prefecture Univ. (Japan)

7986 Ol Third order silicon (Si) nitride side-walled grating using silicon-on- insulatir (SOI) [7986-39]

C. E. Png, S. T. Lim, E. P. Li, A*STAR (Singapore); A. J. Danner, A*STAR (Singapore) and National Univ. of Singapore (Singapore)

7986 0J Modeling of insertion loss of fiber Fabry-Perot filter with a bidirectional beam propagation method [7986-07]

H. Qi, Huazhong Univ. of Science and Technology (China) and Huangshi Institute of Technology (China); Y. Yu, Huazhong Univ. of Science and Technology (China)

7986 OK An optimal design of 25-GHz flat-top multichannel optical filters [7986-31]

M. XU, Communication Univ. of China (China); L. C. Wu, C. C. Li, H. M. Yang, I-Shou Univ. (Taiwan, China) 
7986 OL Birefringence property of asymmetric structure photonic crystal fiber [7986-32]

M. Liu, Handan College (China) and Hebei Univ. of Technology (China); Y. Yue, Hebei Univ. of Technology (China); Y. Li, Handan College (China)

\section{SESSION 8 SIGNAL PROCESSING AND SYSTEM II}

7986 OM Influence of uplink limitation and broadcast traffic on power efficiency in long-reach optical access network [7986-42]

A. Lovrić, S. Aleksić, Vienna Univ. of Technology (Austria)

7986 ON Polarization independent acousto-optic filter based on photonic crystal fibers by using a fiber loop mirror [7986-33]

C.-L. Zhao, China Jiliang Univ. (China); W. Jin, J. Ju, Hong Kong Polytechnic Univ. (Hong

Kong, China); X. Dong, J. Kang, Z. Zhang, S. Jin, China Jiliang Univ. (China)

\section{POSTER SESSION}

798600 Experimental research on preamplifier for high power $\mathrm{Yb}$-doped fiber pulse amplification [7986-02]

F. Liu, Hunan Univ. of Technology (China) and Nankai Univ. (China); X. Guo, N. Li, L. Kong,

H. Wen, C. Li, Hunan Univ. of Technology (China)

7986 OP Tunable flat-top polarization interleaver filters based on cascaded film cavity structure [7986-03]

J. Zhang, S. YU, X. Li, S. Guo, Shanghai Univ. (China)

7986 OQ Design of optical notch filter based on Michelson Gires-Tournois interferometer [7986-10]

S. Guo, J. Zhang, X. Li, Shanghai Univ. (China)

7986 OR Highly birefringent photonic crystal fiber with hybrid cladding structure [7986-11]

J. Li, R. Wang, J. Wang, B. Zhang, H. Zhou, The PLA Univ. of Science and Technology (China)

7986 OS Comparison of $\mathrm{TiO}_{2}$-doped $\mathrm{SiO}_{2}$ films from two organosilicon precursors [7986-12]

J. P. Bange, Gunma Univ. (Japan); L. S. Patil, D. K. Gautam, North Maharashtra Univ. (India)

7986 OT A novel terahertz waveguide: polymer tube [7986-13]

D. Chen, Zhejiang Normal Univ. (China)

7986 OU Research on reflectivity of chemical composition grating sensors at high temperatures [7986-14]

G. Li, Handan College (China); B. Guan, Jinan Univ. (China)

7986 OV All-normal-dispersion dissipative soliton Yb fiber laser [7986-16]

Z. Zhang, P. Liang, Jiangxi Normal Univ. (China) 
7986 OW A SOA-based high Q microwave photonic filter [7986-21]

E. XU, Nanjing Univ. of Posts and Telecommunications (China) and Huazhong Univ. of Science \& Technology (China); L. Li, Nanjing Univ. of Posts and Telecommunications (China);

F. Wang, Y. YU, X. Li, X. Zhang, D. Huang, Huazhong Univ. of Science \& Technology (China)

7986 0X Refraction index measurement using long period grating fabricated by symmetrical-hole fiber [7986-22]

J. Kang, China Jiliang Univ. (China) and Univ. of Shanghai for Science and Technology (China); X. Dong, China Jiliang Univ. (China); M. Li, Univ. of Shanghai for Science and Technology (China); Z. Zhang, S. Jin, China Jiliang Univ. (China)

7986 OY Multiplexed extremely short distributed Bragg reflector fiber laser array for large-scale sensing applications [7986-23]

A. C. L. Wong, W. H. Chung, H. Y. Tam, C. Lu, Hong Kong Polytechnic Univ. (Hong Kong, China)

$79860 Z$ Design of interleavers with fiber ring coupled structure based on digital signal processing theory [7986-24]

X. Li, J. Zhang, S. Guo, Shanghai Univ. (China)

798610 Acrylic-based 1x2 Y-Branch POF coupler with high index contrast waveguide taper [7986-26]

A. A. Ehsan, Univ. Kebangsaan Malaysia (Malaysia); M. K. Abd Rahman, Univ. Teknologi MARA (Malaysia)

798611 Analysis of the stress-optical effects in silica-on-silicon optical waveguides [7986-27] T. Lang, China Jiliang Univ. (China) and Zhejiang Univ. (China); J. Kuang, X. Lin, Zhejiang Univ. (China)

798612 Simple assembly mechanical splicer using stripping-free fiber connection method [7986-28] H. Hirota, Y. Abe, S. Asakawa, J. Kobayashi, NTT Corp. (Japan)

798613 Research on PMD mitigation by using distributed fast polarization scrambling and FEC [7986-38]

D. Han, M. Li, L. Xi, Beijing Univ. of Posts and Telecommunications (China)

798614 Generation of optical pulses using a self-seeded Fabry-Perot laser diode and a tilted multimode fiber Bragg grating [7986-47]

T. Cai, Y. Liu, T. Wang, Shanghai Univ. (China)

798615 Fiber laser sensor based on fiber-Bragg-grating Fabry-Perot cavity [7986-49]

J. Chen, Y. Liu, T. Cai, T. Wang, Shanghai Univ. (China)

798616 The bending sensitivity of long-period fiber gratings written by $\mathrm{CO}_{2}$ laser under external tension [7986-50]

D. Yang, Y. Liu, W. Tu, T. Wang, Shanghai Univ. (China)

798617 Loss effects on properties of two configurations of novel dual coupled microring resonators [7986-52]

X. Zhang, J. Li, F. Pang, Y. Liu, Shanghai Univ. (China) 
798618 Design and analysis of the high-order mode dispersion compensating fiber [7986-65]

G. Lin, X. Dong, S. Juan, Xiamen Univ. (China)

798619 Influence of the splicing on the birefrengence characteristics of the highly birefringent photonic crystal fiber [7986-66]

S. Juan, X. Dong, G. Lin, J. Hao, Xiamen Univ. (China); Z. Shi, Shanghai Univ. (China)

7986 1A Nonclassical light in array of single mode optical waveguides [7986-102]

A. Rostami, A. Javadi, Univ. of Tabriz (Iran, Islamic Republic of)

Author Index 
Downloaded From: https://www.spiedigitallibrary.org/conference-proceedings-of-spie on 26 Apr 2023

Terms of Use: https://www.spiedigitallibrary.org/terms-of-use 


\title{
Symposium Committees
}

\author{
General Chairs
}

Weishing Hu, Shanghai Jiao Tong University (China)

Ming-Jun Li, Corning, Inc. (United States)

Dennis L. Matthews, University of California, Davis (United States)

Technical Program Chairs

Dominique Chiaroni, Alcatel-Lucent (France)

Jian-Jun He, Zhejiang University (China)

Ken-ichi Kitayama, Osaka University (Japan)

Xingde Li, Johns Hopkins University (United States)

Local Organizing Committee

Nan Chi (Chair), Fudan University (China)

Chansai Cheng, Pudong Optoelectronics Industry Association of

Shanghai (China)

Meiyu Cao, Pudong Optoelectronics Industry Association of Shanghai (China)

Yaohui Jin, Shanghai Jiao Tong University (China)

Ronghui Qu, Shanghai Institute of Optics and Fine Mechanics (China)

Feng Huang, Alcatel-Lucent Shanghai Bell (China)

Organizing Committee

Lirong Zheng, Fudan University (China)

Liangyao Chen, Fudan University (China)

Yiwei Shi, Fudan University (China)

Bo Hu, Fudan University (China)

Rongjun Zhang, Fudan University (China)

Zhengcai Bian, Fudan University (China)

Jianjun Yin, Fudan University (China)

Xinyue Guo, Fudan University (China)

Xiaolu Wan, Fudan University (China)

General Secretariat

Yufeng Shao, Fudan University (China) 
Steering Committee Chairs

Kwok-Wai Cheung, Chinese University of Hong Kong (Hong Kong, China)

Thomas Koch, Lehigh University (United States)

John Zyskind, Mintera Corporation (United States)

Steering Committee Members

Connie Chang-Hasnain, University of California, Berkeley (United States)

Chongcheng Fan, Tsinghua University (China)

Sailing He, Zheijang University (China)

Peter Kaiser, Telcordia Technologies (retired) (United States)

Thomas Koch, Lehigh University (United States)

Ming-Jun Li, Corning, Inc. (United States)

Tingye Li, AT\&T Laboratories (retired) (United States)

Mao Qian, WRI-Fiberhome (China)

Xiaomin Ren, Beijing University of Posts and Telecommunications (China)

Bingkun Zhou, Tsinghua University (China) 


\title{
Conference Committee
}

\author{
Conference Chair \\ Perry P. Shum, Nanyang Technological University (Singapore) \\ Cochairs \\ John H. Marsh, University of Glasgow (United Kingdom) \\ Tanya M. Monro, The University of Adelaide (Australia) \\ Bishnu P. Pal, Indian Institute of Technology Delhi (India) \\ Session Chairs \\ Signal Processing and System I \\ Alan Pak Tao Lau, Stanford University (United States) \\ Nano Fiber \\ Bai-Ou Guan, Jinan University (China) \\ Best Student Paper Session \\ Perry P. Shum, Nanyang Technological University (Singapore) \\ Fiber Laser \\ Yoonchan Jeong, University of Southampton (United Kingdom) \\ Perry P. Shum, Nanyang Technological University (Singapore) \\ Optical Waveguide \\ Lena Wosinski, Royal Institute of Technology (Sweden) \\ Filters and Gratings \\ Wolfgang Sohler, Universität Paderborn (Germany) \\ Specialty Fiber Design and Fabrication \\ Xin Chen, Corning, Inc. (United States) \\ Signal Processing and System II \\ Le N. Binh, Monash University (Australia)
}


Downloaded From: https://www.spiedigitallibrary.org/conference-proceedings-of-spie on 26 Apr 2023

Terms of Use: https://www.spiedigitallibrary.org/terms-of-use 